\title{
Comparative analysis of annexin-1 in neuroepithelial tumors shows altered expression with the grade of malignancy but is not associated with survival
}

\author{
Jens Schittenhelm ${ }^{1}$ Katrin Trautmann ${ }^{1}$, Ghazaleh Tabatabai ${ }^{2}$, Caroline Hermann ${ }^{2}$, \\ Richard Meyermann ${ }^{1}$ and Rudi Beschorner ${ }^{1}$ \\ ${ }^{1}$ Institute of Brain Research, University of Tuebingen, Tuebingen, Germany and ${ }^{2}$ Hertie Institute of Neurology, \\ University of Tuebingen, Tuebingen, Germany
}

\begin{abstract}
In various types of cancer, the expression of members of the annexin family of calcium- and phospholipidbinding anti-inflammatory proteins is dysregulated. Annexin-1 (ANXA1, lipocortin-1) is involved in proliferation, differentiation and apoptosis. It serves as a substrate for the epidermal growth factor receptor (EGFR), which is frequently amplified in primary gliomas. It is unclear how annexin-1 is expressed in various neuroepithelial tumors, and whether there is any association with tumor malignancy or survival. We studied annexin-1 expression in 394 glial neoplasms of all grades of malignancy and 81 normal brain samples by immunohistochemistry using tissue microarrays. The results were validated using western blot and reverse transcription-PCR (RT-PCR). In the normal human brain, the expression of annexin-1 is limited to ependymal cells and subependymal astrocytes, but is also upregulated in reactive astrocytes. Ependymomas and astrocytomas showed significantly higher mean annexin-1 expression levels in the cytoplasm compared with oligodendrogliomas (both: $\boldsymbol{P}<\mathbf{0 . 0 0 0 1 )}$. In addition, nuclear staining of annexin-1 in oligodendroglial tumor cells was significantly reduced $(P=0.0002)$, which may be used as a diagnostic tool for differentiating between astrocytomas and oligodendrogliomas. Although annexin-1 expression in ependymomas decreased with the grade of malignancy, diffuse astrocytomas showed a significant increase in cytoplasmic annexin-1-positive tumor cells. However, survival analysis showed that the expression of annexin-1 is not associated with patient survival. Similar to the EGFR amplification profile, primary glioblastomas had a higher annexin-1 expression level compared with secondary glioblastomas. Thus, annexin-1 upregulation in astrocytomas may contribute to tumor progression and its expression profile is similar to its substrate, EGFR, suggesting a possible regulation thereof.
\end{abstract}

Modern Pathology (2009) 22, 1600-1611; doi:10.1038/modpathol.2009.132; published online 18 September 2009

Keywords: Annexin-1; glioma; astrocytoma; oligodendroglioma; ependymoma; central nervous system

Annexin-1 (ANXA1, lipocortin-1) belongs to a structurally related family of calcium-binding ('annexing') anti-inflammatory proteins that act extracellularly as a strong inhibitor of eicosanoid synthesis and phospholipase A2. ${ }^{1}$ Annexins have one phospholipid-binding domain and an amino terminal domain that determine their specificity. ${ }^{2}$

Correspondence: Dr J Schittenhelm, MD, Institute of Brain Research, University of Tuebingen, Calwerstr. 3, Tuebingen D72076, Germany.

E-mail: jens.schittenhelm@med.uni-tuebingen.de

Received 20 April 2009; revised 8 July 2009; accepted 21 July 2009; published online 18 September 2009
The cytosolic $37 \mathrm{kDa}$ protein annexin- 1 is found especially in the neutrophils, lung, intestine, skin and seminal fluid, ${ }^{3}$ where it is involved in differentiation of both glandular and squamous epithelia. ${ }^{1}$ In the normal human brain, it is expressed in ependymal cells and microglia. ${ }^{4}$ Results regarding its expression in neurons and astrocytes are controversial, ${ }^{4,5}$ while oligodendroglial cells are generally spared. ${ }^{6}$ In pathological conditions, annexin-1 is also expressed in the macrophages, perivascular lymphocytes and reactive astrocytes..$^{5,8}$ There is evidence that intracellular annexin-1 is involved in proliferation, differentiation and apoptosis. ${ }^{8-11}$ The expression varies among different types of 
Table 1 An overview of patients' data

\begin{tabular}{|c|c|c|c|}
\hline Diagnosis & WHO grade & $\begin{array}{l}\text { Mean age } \\
\text { (range) }\end{array}$ & $\begin{array}{c}\text { Number of samples } \\
\text { (female/male/unknown) }\end{array}$ \\
\hline Pilocytic astrocytoma & $\mathrm{I}$ & $20.5(3-56)$ & $32(12 / 20)$ \\
\hline Diffuse astrocytoma & II & $43.7(18-77)$ & $92(36 / 51 / 5)$ \\
\hline Anaplastic astrocytoma & III & $48.1(19-76)$ & $68(25 / 38 / 5)$ \\
\hline Glioblastoma & IV & $59.9(25-85)$ & $92(42 / 50)$ \\
\hline Oligodendroglioma & II & $42.6(3-66)$ & $29(12 / 17)$ \\
\hline Anaplastic oligodendroglioma & III & $50.5(23-75)$ & $23(14 / 9)$ \\
\hline Myxopapillary ependymoma & I & $26.4(10-46)$ & $5(1 / 4)$ \\
\hline Ependymoma & II & $31.3(3-68)$ & $11(2 / 9)$ \\
\hline Anaplastic ependymoma & III & $52(2-78)$ & $4(1 / 3)$ \\
\hline Ganglioglioma & I-III & $27.1(8-46)$ & $10(5 / 5)$ \\
\hline Dysembryoplastic neuroepithelial tumor & $\mathrm{I}$ & $7.6(4-14)$ & $5(3 / 2)$ \\
\hline Central neurocytoma & II & $44.3(20-73)$ & $4(2 / 2)$ \\
\hline Pilomyxoid astrocytoma & II & $23(16-31)$ & $3(2 / 1)$ \\
\hline Pleomorphic xanthoastrocytoma & II & $36.5(13-60)$ & $2(0 / 2)$ \\
\hline Subependymal giant cell astrocytoma & $\mathrm{I}$ & $34.3(26-39)$ & $4(2 / 2)$ \\
\hline Subependymoma & I & $55.5(44-61)$ & $4(3 / 1)$ \\
\hline Central nervous system primitive neuroectodermal tumor & IV & $50.5(46-55)$ & $2(0 / 2)$ \\
\hline Medulloblastoma & IV & $26.5(5-60)$ & $8(5 / 3)$ \\
\hline Choroid plexus papilloma & $\mathrm{I}$ & $39(32-57)$ & $4(0 / 4)$ \\
\hline Normal brain and spinal cord (adult) & & $52.1(1-92)$ & $81(12 / 17)$ \\
\hline Normal brain (fetal) & & 17-40th g.w. & 54 (ND) \\
\hline Pathologic brain (gliosis) & & $58.1(37-80)$ & $14(5 / 9)$ \\
\hline
\end{tabular}

ND, not documented; g.w., = gestation week.

cancer, as there is reduced expression in the lymphomas, esophageal, head and neck and prostrate cancers, whereas upregulated expression is observed in the pituitary, skin, breast and bladder tumors (reviewed by Lim and Pervaiz ${ }^{1}$ ). One of the many different functions of annexin-1 is activation of the mitogen-activated protein kinase/extracellular signal-regulated kinase (MAPK/ERK) pathway, independent of nuclear factor- $\kappa \mathrm{B},{ }^{9,12}$ thereby acting as a substrate for the epidermal growth factor receptor (EGFR) and Src-family tyrosine kinases, including protein kinase C. ${ }^{13-15}$

A majority of neuroepithelial tumors are of glial origin and are derived from the astrocytes, oligodendroglia, ependymal cells or their precursors. These are classified according to the WHO criteria in a four-tiered system. ${ }^{16}$ The most malignant tumor is the glioblastoma that arises either in approximately $90 \%$ de novo or secondary in $10 \%$ by progression of lower-grade astrocytomas. ${ }^{17}$ On a molecular level, these tumors differ by EGFR gene amplification and associated EGFR receptor overexpression in primary glioblastomas. ${ }^{18}$ As annexin-1 is a known substrate for EGFR, we studied the expression of annexin-1 in various neuroepithelial tumors to determine annexin-1 expression levels dependent on the tumor WHO grade and with regard to a possible application of annexin-1 as a diagnostic marker in routine immunohistochemistry.

\section{Materials and methods}

\section{Patient Data}

The brain tumor samples enrolled in this study were retrieved from the tumor bank of the Institute of
Brain Research, University of Tübingen, as previously reported. ${ }^{19}$ The usage of human specimens was in accordance with the University's ethics commission. The samples included 52 oligodendrogliomas, 20 ependymomas and 284 astrocytomas. The epidemiological data of the patients are given in Table 1. Twenty-nine autopsy cases (consisting of 81 samples from different regions) belonging to an established normal brain bank, 14 autopsy cases with known gliotic changes due to various pathologic conditions (hypoxia, infarction, etc) and fetal brain tissue from 21 autopsy cases (consisting of 54 samples from different regions) were also included. In addition, tumor samples of gangliogliomas, central neurocytomas, pleomorphic xanthoastrocytomas, pilomyxoid astrocytomas, medulloblastomas, subependymomas, subependymal giant cell astrocytomas, choroid plexus papillomas and dysembryoplastic neuropithelial tumors were included in the study (details are given in Table 1). Histological diagnosis and grading for each tumor sample was performed according to the current WHO classification of tumors of the central nervous system by at least two, and in most cases three, experienced neuropathologists. ${ }^{16}$

\section{Tissue Microarray}

Tissue microarrays were constructed by extracting cylindrical tissue core biopsies showing representative tissue sections from the paraffin donor blocks followed by re-embedding these into a pre-punched hole on a single recipient paraffin block at defined array coordinates. Using this technique, previously 
selected tumor regions were extracted and prepared as a tissue microarray. A tissue microarray machine (Beecher Instruments, Inc., Sun Prairie, WI, USA) was used to extract a $600-\mu \mathrm{m}$ cylindrical core sample from the tissue donor block. The tissue microarray blocks were then cut with a microtome (3- $\mu \mathrm{m}$ thickness) and placed on SuperFrost Plus slides (Microm International, Walldorf, Germany).

\section{Immunohistochemistry}

Immunohistochemistry with anti-annexin-1 antibody was performed on formalin-fixed paraffinembedded tissue sections (dilution 1:1000, BD Transduction Labs, San Diego, CA, USA). The sections were deparaffinized and immunostained using a standard protocol on the Benchmark immunohistochemistry system (Ventana Medical Systems, Tucson, AZ, USA). In brief, the method uses an indirect biotin-avidin system and an universal biotinylated immunoglobulin secondary antibody and diaminobenzidine as substrate. Afterward, the sections were incubated with a copper enhancer (Ventana) and counterstained with hematoxylin. Human skin or kidney samples served as positive controls. Negative control slides were processed in parallel with each batch of staining by replacing the use of primary antibody with dilution buffer.

\section{Western Blot}

Tumor lysates from 18 snap-frozen tissue were prepared in $10 \mathrm{mM}$ Tris-HCl (pH 7.2) containing $50 \mathrm{mM} \mathrm{NaCl}, 5 \mu \mathrm{M} \mathrm{ZnCl}, 2 \mathrm{mM}$ dithiothreitol, 30, $\mathrm{mM}$ sodiumpyrophosphate, 1 vol\% Triton X-100. Protease inhibition was performed using the Complete Mini Inhibitor, EDTA-free system (Roche, Mannheim, Germany). After normalization, aliquots of lysates were electrophoresed on $8-12 \%$ SDSPAGE gels under reducing conditions and transferred to nitrocellulose (Schleicher \& Schuell, Dassel, Germany). Equal protein loading was ascertained using Ponceau S staining. After blocking nonspecific-binding sites with $5 \%(\mathrm{w} / \mathrm{v})$ dried milk in PBS for $30 \mathrm{~min}$, the filters were incubated with specific primary antibodies overnight at $4^{\circ} \mathrm{C}$, washed and incubated with horseradish peroxidase-conjugated donkey anti-goat IgG or goat antimouse IgG (diluted 1:1000) (Santa Cruz Biotechnology, Santa Cruz, CA, USA) for $3 \mathrm{~h}$ at $22^{\circ} \mathrm{C}$. The primary antibodies sc-1616 goat anti-beta-actin (Santa Cruz Biotechnology), mouse anti-annexin-1 (BD Transduction Labs), were diluted 1:5000 in phosphate-buffered saline containing $0.05 \%$ Tween20 and $5 \%$ skim milk. Protein bands were visualized using enhanced chemiluminescence (Amersham, Braunschweig, Germany) and quantified using Image J software (NIH, Bethesda: http://rsb.info. nih.gov/ij). Annexin-1 expression levels were then normalized in relation to $\beta$-actin expression for each sample.

\section{RT-PCR}

Total RNA from eight tumors was isolated using the RNeasy kit (Qiagen, Hildesheim, Germany), cDNA was generated using MMLV reverse transcriptase (Promega) according to the manufacturer's instructions and amplification for annexin-1 was carried out using standard protocols. The conditions for all PCRs were 30 cycles at $95^{\circ} \mathrm{C}$ for $30 \mathrm{~s}$ and at $58^{\circ} \mathrm{C}$ for $45 \mathrm{~s}$ followed by $1 \mathrm{~min}$ at $72^{\circ} \mathrm{C}$. The used primers were: forward $5^{\prime}$-GCAGGCCTGGTTTATTGAAA- $3^{\prime}$ and reverse 5'-GCTGTGCATTGTTTCGCTTA- $3^{\prime}$, yielding a product of $203 \mathrm{bp}$. PCRss were performed on an Eppendorf Thermocycler (Eppendorf, Hamburg, Germany) using aTaq polymerase (Promega) according to the manufacturer's instructions.

\section{Evaluation and Statistics}

Annexin-1 expression in endothelial vessels served as an internal positive control. Cytoplasmic expression of annexin-1 in tumors was evaluated using a semiquantitative score as 0 (no staining), 1 (singular positive cells, <1\%), $2(2-10 \%), 3(11-20 \%), 4(21-$ $50 \%)$ and $5(>50 \%)$ independently by two raters (Figure 1). Contingency analysis for annexin-1 score followed by Mantel-Haenszel chi-square test was performed for dependency on WHO grade or patient's gender. Ordinal logistic regression was performed to test whether or not annexin-1 expression was age dependent. To test inter-observer variability in the annexin-1 assessment, a marginal homogeneity test for symmetry in a contingency table was calculated. The $\kappa$ values of $0-0.20$ were defined as slight, $0.21-0.40$ as fair, $0.41-0.60$ as moderate, $0.61-0.80$ as substantial and $0.81-1.0$ as almost perfect. ${ }^{20}$ Survival analysis was performed with the Kaplan-Meier curves and significance determined using log-rank test for selected groups of annexin-1-positive tumors and nuclear staining stratified for WHO grade. JMP 7.0 was used for statistical analysis.

\section{Results}

\section{The Applied Annexin-1 Scoring Method is an Easy and Reproducible Method}

The inter-observer variability for all cases examined differed at most by two scores (1/455 differed by two scores and 56/455 differed by one score). Statistical analysis showed that the agreement with regard to annexin-1 scores between raters was almost perfect $(\kappa=0.824)$. Only five cases differed between score 0 (no expression) and score 1 (single cells positive) and were re-evaluated for consensus diagnosis. 

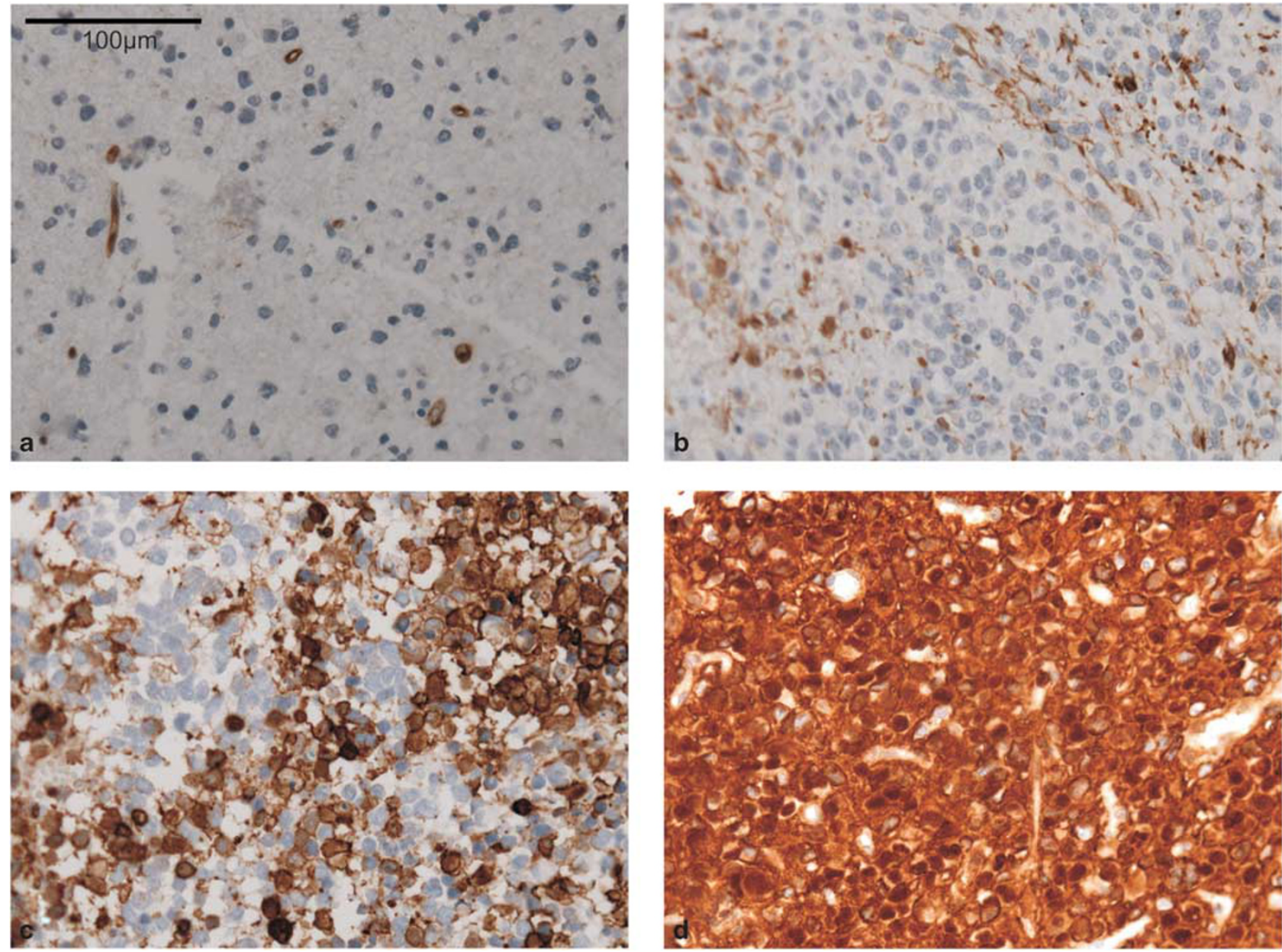

Figure 1 Representative staining scores: (a) score 1, single positive cells, (b) score 2 (2-10\% positive cells), (c) score 4 (21-50\% positive cells) and (d) score 5 (>50\% positive cells).

\section{In Normal Human Brains, Annexin-1 is Expressed in Ependymal Cells as Well as in Subependymal Astrocytes}

In normal central nervous system tissue specimens of the cortex, grey (Figure 2a) and white matter (Figure 2b) annexin-1 expression was limited to endothelial cells of small capillaries and vessels, but was absent in neurons, astrocytic or oligodendroglial cells. Similar observations were made in the hippocampus, thalamus, pallidum, putamen, mesencephalon, cerebellum, pons and medulla oblongata. In contrast, expression of annexin-1 was observed in ependymal cells lining the lateral, third and fourth ventricles and in the subependymal astrocytes (Figure 2c). In the spinal cord, the ependymal lining of the central canal and subependymal astrocytes were also immunoreactive for annexin-1 (Figure 2d). Occasionally, few epithelial cell membranes of the choroid plexus were also immunoreactive for annexin-1. In various pathological conditions (hypoxia, infarction or brain metastasis), the surrounding central nervous system tissue with astrogliosis showed upregulation of annexin-1 in microglial cells and reactive astrocytes (Figure 2e), although the numbers of annexin1-positive glia were lower than those expressing GFAP. In addition, some macrophages surrounding infarction were also immunoreactive for annexin-1. In fetal brains, annexin-1 was also present in endothelial cells of small capillaries. Expression of annexin-1 was found in the ependymal layer lining lateral and fourth ventricles, as well as a few neuronal processes in the germinal matrix (Figure 2f). No immunoreactivity was detectable in the neurons, astrocytes or oligodendrocytes. In contrast to adult specimens, fetal choroid plexus epithelium constantly lacked annexin-1.

\section{Annexin-1 is Expressed in Various Levels in Human Gliomas In Vivo}

Western blot showed a distinct lane at $38 \mathrm{kDa}$ in all 18 glioma samples examined (Figure 3a). The intensity varied from 0.5 -fold up to sevenfold overexpression in relation to $\beta$-actin intensity. The highest relative expression levels were observed in 

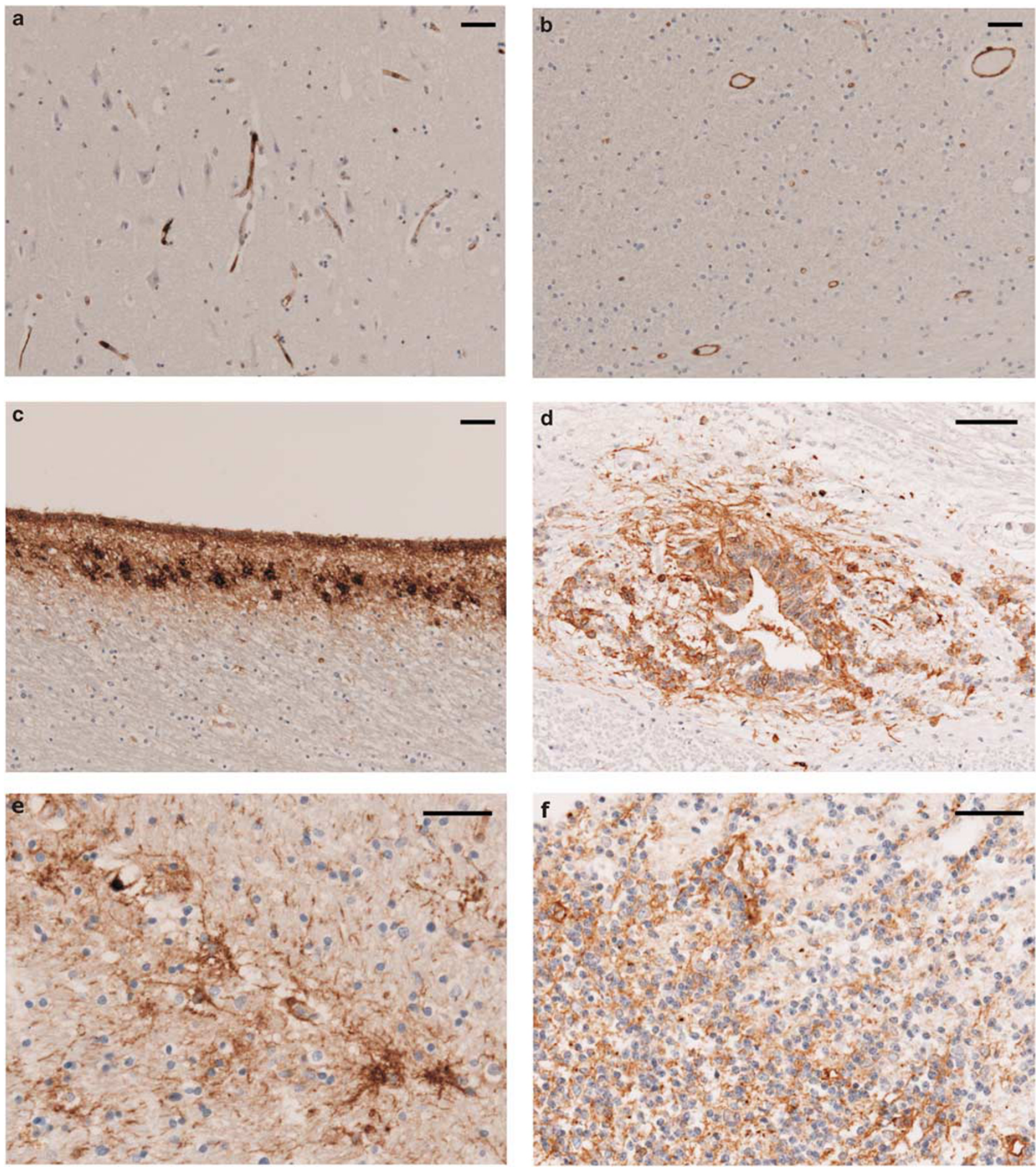

Figure 2 Annexin-1 expression in the human brain. (a) grey matter; (b) white matter; (c) ependymal lining and subependymal astrocytes of the lateral ventricle; (d) ependymal lining of the central canal; (e) reactive gliosis; and (f) fetal brain. Note the constitutive expression of annexin-1 in endothelial cells in a, b and f. scale bars a-f: $50 \mu \mathrm{m}$.

glioblastoma specimens (mean: fourfold), whereas anaplastic astrocytoma (mean: twofold) and pilocytic astrocytoma (mean 2.3-fold) showed even higher relative expression levels than oligodendroglial neoplasms (grade II WHO: 0.7-fold; grade III WHO 0.8-fold). Simple linear regression analysis showed a strong correlation between intensity of all 18 western blot lanes normalized to glyceraldehyde-3phosphate dehydrogenase (GAPDH) and immunohistochemistry score of each sample $(P=0.008)$. This validates the established annexin- 1 immunohistochemistry score for protein quantification. The analysis of immunohistochemistry data showed a significant upregulation $(P<0.001)$ of annexin-1 in 


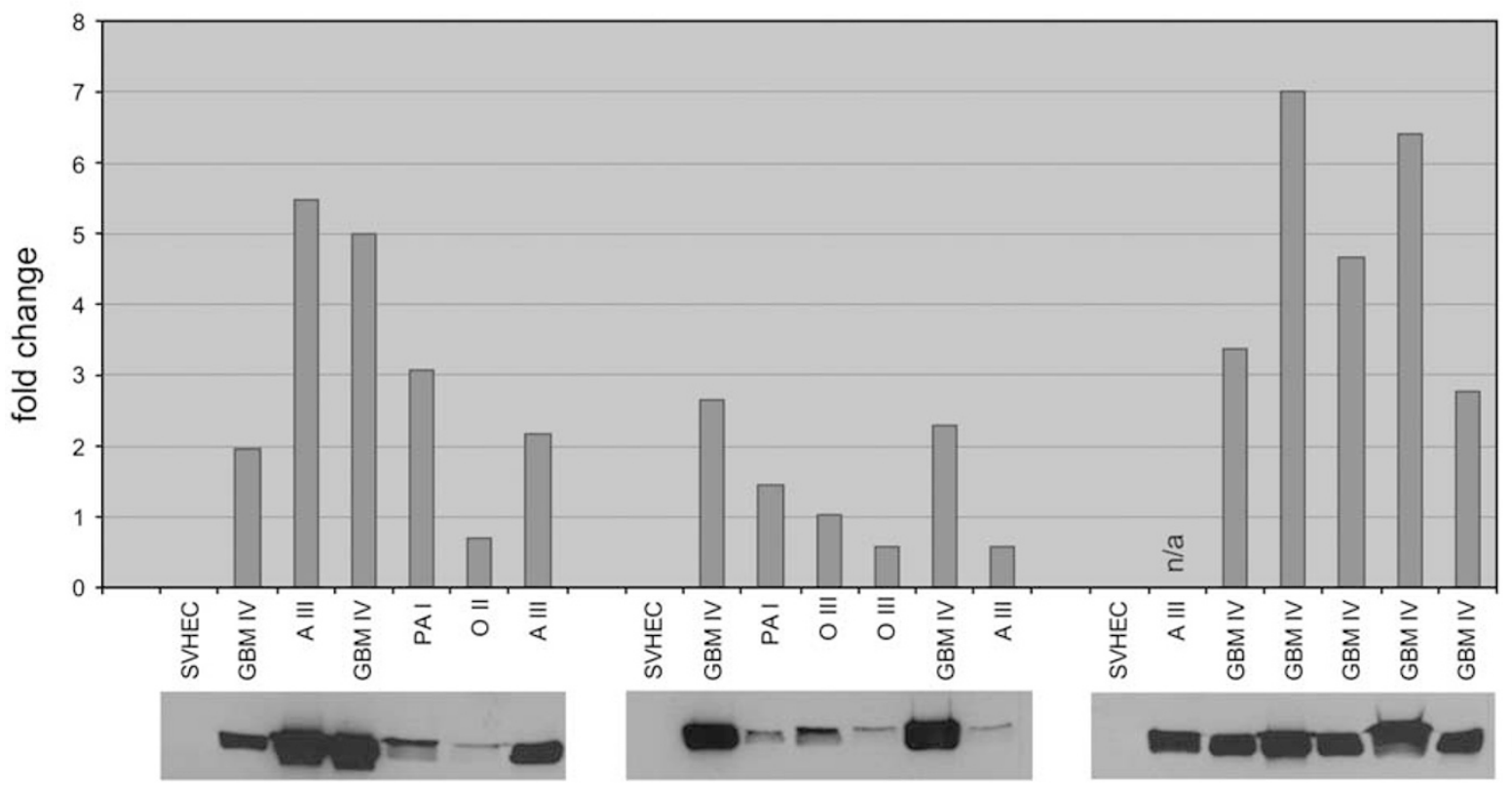

$38 \mathrm{kDa}$

b RT-PCR

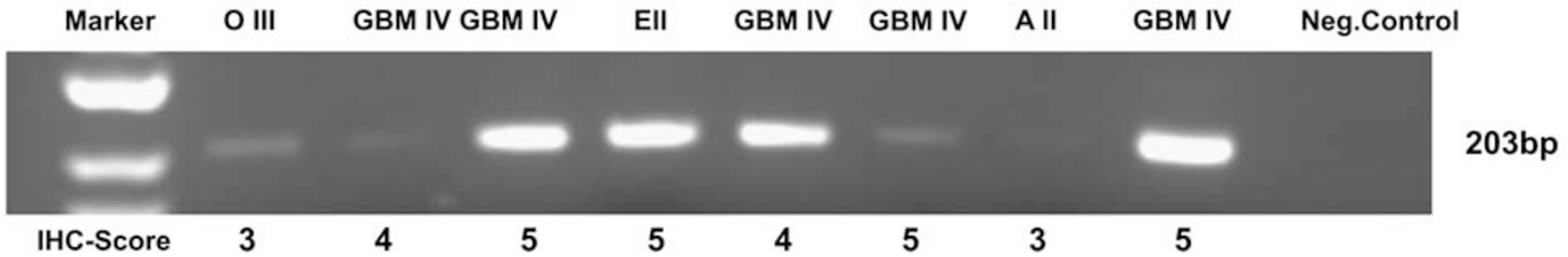

Figure 3 (a) Western blot showing annexin-1 expression relative to GAPDH in various gliomas. There is a significant correlation with the immunohistochemical expression score $(P=0.0008)(b)$ : reverse transcriptase-PCR results in selected gliomas in relation to the immunohistochemistry results (IHC score) that are depicted below.

all gliomas $(n=356)$ compared with normal brain white and grey matter $(n=72$; mean: $0.1 ; 95 \%$ confidence interval (CI): 0.04-0.2). The highest annexin-1 expression levels were observed in ependymomas ( $n=20$; mean: 4.1; 95\% CI: 3.3-4.8), followed by astrocytomas of all grades $(n=284$; mean: 2.9 ; 95\% CI: 2.8-3.2) and lowest levels in oligodendrogliomas $(n=52$; mean: 1.6; 95\% CI: $1.4-$ 1.8). Significant differences in mean expression levels were observed not only for ependymoma when compared with astrocytoma $(P=0.0043)$ and oligodendroglioma $(P<0.0001)$, but also for astrocytoma when compared with oligodendroglioma $(P<0.0001)$.

\section{RT-PCR Confirms Annexin-1 Expression in Gliomas}

Reverse transcription-PCR using a primer pair at positions 101 and 303 of the annexin-1 mRNA yielded a product of the expected size of $203 \mathrm{bp}$ (Figure 3b) in all eight glial neoplasms examined. The strongest signal was observed in an ependymoma and in three out of five glioblastoma samples. All tumors had an immunohistochemical score of 3 or higher (ie, at least $11 \%$ immunoreactive tumor cells). Although correlation with immunohistochemistry score was only moderate, RT-PCR data confirmed that mRNA expression of annexin-1 is present in gliomas.

\section{Annexin-1 Expression in Diffuse Astrocytic Neoplasms Increases with the Grade of Malignancy}

After the exclusion of recurrent tumors, 276 astrocytomas from different WHO grades (WHO I-IV) were analyzed in detail. In total, $82 \%(226 / 276)$ astrocytomas contained annexin-1-positive tumor cells, with the majority of immunonegative tumors being diffuse astrocytomas grade II WHO $(n=31$; $34 \%$, Figure $4 \mathrm{~b}$ ). In astrocytomas, annexin-1 was distributed in the perinuclear cytoplasm and in the nuclei in the majority of tumors (Table 2). Controlling for each WHO grade, there was no association between cytoplasmic annexin-1 expression and the patient's gender. Although there was a slight trend for increased annexin-1 expression levels with age 
a

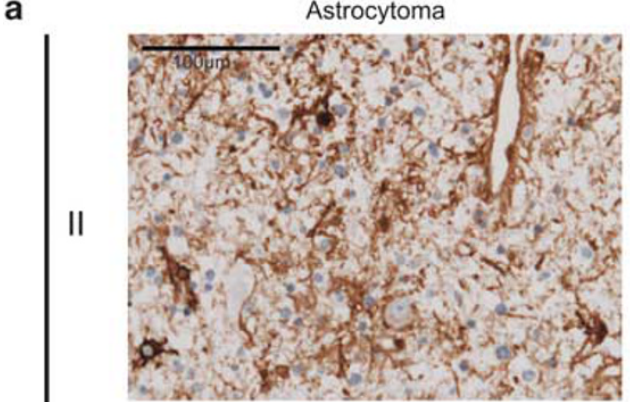

III

IV

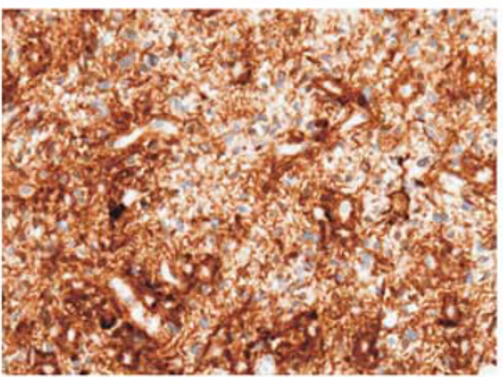

I
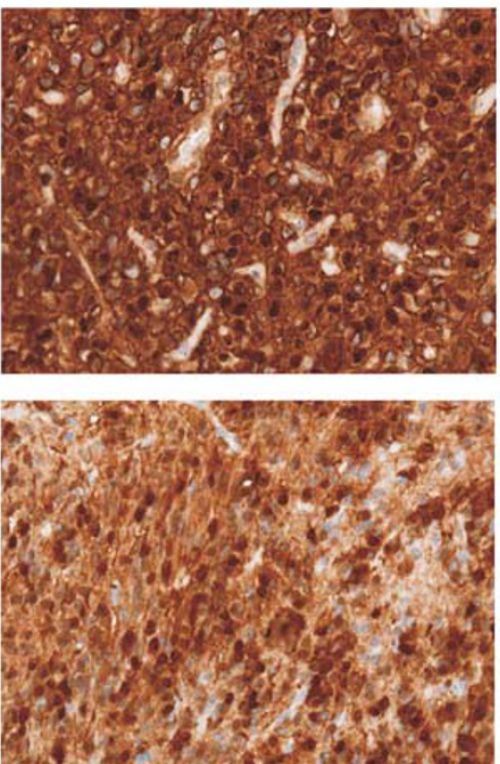

Oligodendroglioma
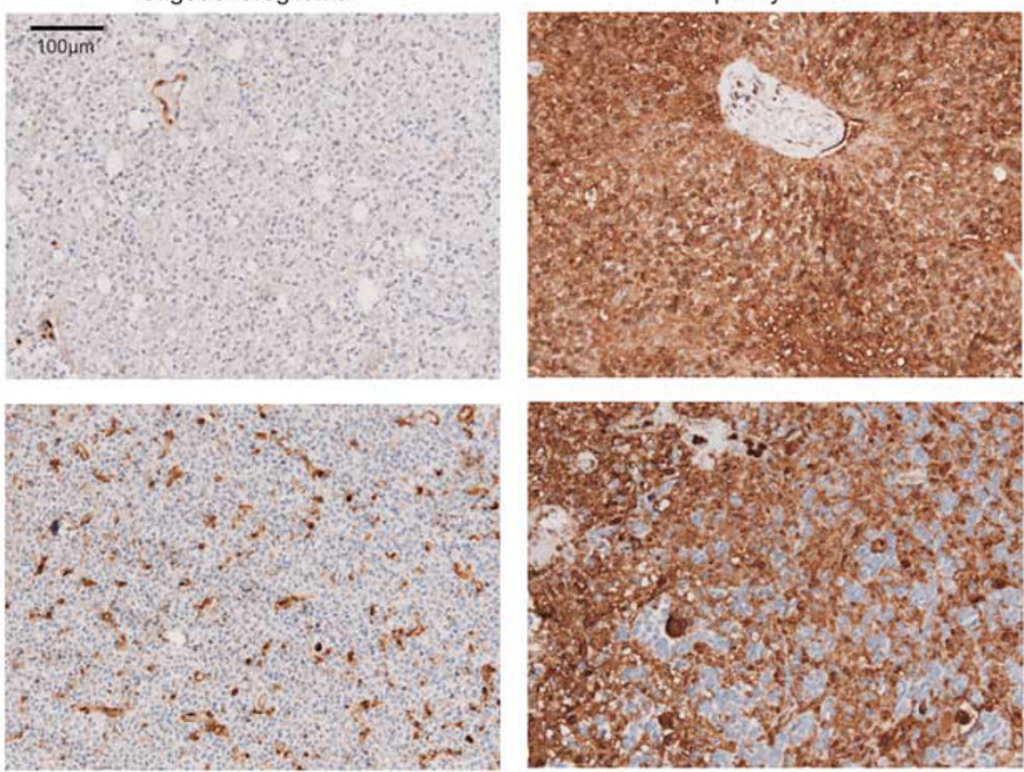

b

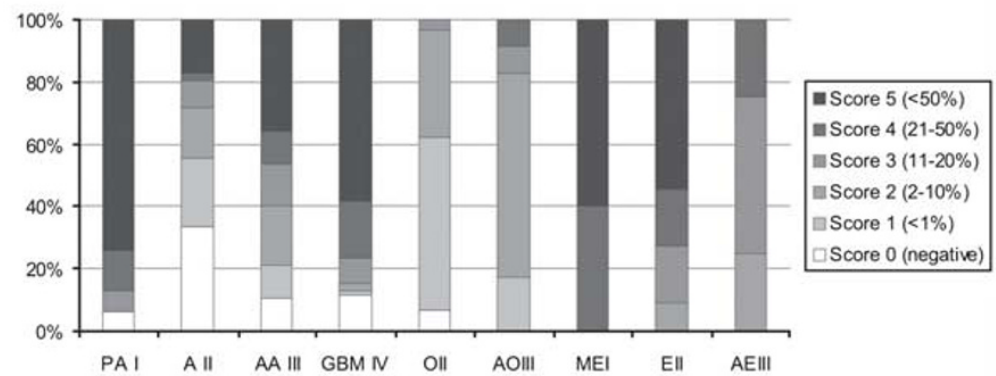

Figure 4 (a) Representative immunohistochemical staining results for annexin-1 in astrocytomas (left), oligodendrogliomas (middle) and ependymomas (right column) of the different WHO grades. (b) Each graph provides the distribution of tissue cores scored as negative (score 0 , white) to more than 50\% immunoreactive tumor cells (score 5, black). PA I, pilocytic astrocytoma grade I WHO; A II, diffuse astrocytoma grade II WHO; AA III, anaplastic astrocytoma grade III WHO; GBM IV, glioblastoma grade IV WHO; O II, oligodendroglioma grade II WHO; AO III, anaplastic oligodendroglioma grade III WHO; ME I, myxopapillary ependymoma grade I WHO; E II, ependymoma grade II WHO; and AE III, anaplastic ependymoma grade III WHO.

Table 2 Statistical analysis of immunohistochemistry results

\begin{tabular}{|c|c|c|c|c|}
\hline Tumor (grade) & $\begin{array}{l}\text { Gender } \\
\text { (Mantel-Haenszel) }\end{array}$ & $\begin{array}{l}\text { Age } \\
\text { (logistic fit) }\end{array}$ & $\begin{array}{l}\text { Immunoreactive tumors } \\
\text { (score } 1 \text { or higher) }\end{array}$ & $\begin{array}{l}\text { Nuclear } \\
\text { staining }\end{array}$ \\
\hline Pilocytic astrocytoma I & $P=0.971$ & $P=0.571$ & $29 / 31(94 \%)$ & $10 / 25(40 \%)$ \\
\hline Diffuse astrocytoma II & $P=0.558$ & $P=0.068$ & $61 / 92(66 \%)$ & $24 / 78(31 \%)$ \\
\hline Anaplastic astrocytoma III & $P=0.556$ & $P=0.096$ & $60 / 67(90 \%)$ & $35 / 52(67 \%)$ \\
\hline Glioblastoma IV & $P=0.649$ & $P=0.261$ & $76 / 86(88 \%)$ & $41 / 67(61 \%)$ \\
\hline Oligodendroglioma II & $P=0.263$ & $P=0.912$ & $27 / 29(94 \%)$ & $3 / 27(11 \%)$ \\
\hline Anaplastic oligodendroglioma III & $P=0.051$ & $P=0.253$ & $23 / 23(100 \%)$ & $3 / 19(16 \%)$ \\
\hline Ependymoma II & $P=0.563$ & $P=0.088$ & $11 / 11(100 \%)$ & ND \\
\hline Anaplastic Ependymoma III & $P=0.606$ & $P=0.725$ & $4 / 4(100 \%)$ & ND \\
\hline
\end{tabular}


in WHO grade II and III tumors, the results were not significant (Table 2). The pilocytic astrocytomas, WHO grade I, showed the highest mean annexin-1 expression levels of all the tumors examined (Figure 4; mean 4.5; 95\% CI: 3.9-5.0). The lowest annexin-1 expression levels were present in WHO grade II diffuse astrocytomas (mean 1.7; 95\% CI: 1.3-2.1), with a predominantly perivascular staining pattern of cytoplasmic processes. In WHO grade III anaplastic astrocytomas (mean 3.0; 95\% CI: 2.6-3.4), a significant upregulation $(P=0.0019)$, compared with WHO grade II astrocytomas, was observed. Finally, the highest (mean 3.9; 95\% CI: 3.5-4.3) expression levels were found in WHO grade IV glioblastoma $(P<0.0001$ in comparison with WHO grade II and $P=0.0003$ in comparison with WHO grade III). In glioblastomas, the annexin-1 expression pattern was heterogeneous. In the necrotic areas, annexin-1 immunoreactivity was observed in cells with macrophagic phenotype. Sometimes a slight upregulation in the perinecrotic tumor cells was present, but this was not a constant feature. Most endothelial cells of proliferating vessels in glioblastomas were devoid of annexin-1 staining but occasionally some cells showed nuclear staining. The inner luminal side also showed a weak cytoplasmic immunoreactivity for annexin-1 in some neoplastic vessels.

\section{Annexin-1 Expression in Oligodendrogliomas and Ependymomas}

Next, we examined 52 oligodendrogliomas and 20 ependymomas of different WHO grades of malignancy (WHO I-III) using immunohistochemistry. In oligodendrogliomas, the overall expression levels of annexin-1 were lower than in astrocytomas, typically sparing the honeycomb-like tumor cells (Figure 4a). Focally, few interdispersed tumor cells showed cytoplasmic immunoreactivity in addition to endothelial and macrophagic/microglial cells, the latter also occasionally showing a nuclear staining. Similiar to astrocytic tumors, mean annexin-1 expression level increased significantly $(P=$ 0.0013) from oligodendroglioma grade II WHO (mean: 1.3; 95\% CI: 1.0-1.6) to oligodendroglioma grade III WHO (mean: 2.0; 95\% CI: 1.7-2.3). The presence of nuclear annexin-1-positive cells distinguished astrocytomas and anaplastic oligodendrogliomas with 95\% sensitivity and 26\% specificity and was associated with positive and negative predictive values of 50 and $87 \%$, respectively. Considering a cutoff value of $7 \%$ for annexin-1positive nuclei within each tumor sample, the tumors were distinguished with 100\% sensitivity and $100 \%$ negative predictive value. In contrast to the previous entities, ependymomas showed a decrease in the mean expression levels from WHO grade I myxopapillary ependymoma (mean: 4.8; 95\% CI: 4.5-5.0) over WHO grade II ependymoma (mean: $4.1 ; 95 \%$ CI: $3.3-4.8$ ) to WHO grade III anaplastic ependymoma (mean: 3.0 ; 95\% CI: $1.7-$ 4.3), although the differences were significant only between grade I and grade III WHO $(P=0.0103)$. None of the ependymomas were immunonegative for annexin-1 (Figure 4, Table 2).

\section{Annexin-1 Expression in Primary Glioblastomas is Higher Compared with Secondary Tumors}

Next, we compared annexin-1 expression in 66 primary and 28 secondary glioblastomas. A significant difference was observed $(P=0.0098)$ in primary (de novo) glioblastomas, which had a higher mean expression (mean: 4.8; 95\% CI: 4.2-5.0), compared with secondary glioblastomas (mean: 3.9 ; 95\% CI: 3.4-4.4). In 80 astrocytomas we were able to compare annexin-1 expression scores in the tumor center with diffuse infiltration border as defined previously. ${ }^{21}$ The mean annexin-1 expression levels in the tumor center $(3.6 ; 95 \%$ CI: 3.2-4.1) were higher, compared with the infiltration zone (mean: 1.9; 95\% CI: 0.7-3.2; $P=0.0146)$. There was no significant survival difference in annexin-1-positive and annexin-1-negative tumors (Figure 5), although there was a trend for worse survival in annexin-1negative glioblastomas.

\section{Nuclear Annexin-1 Staining Predominates in Astrocytomas}

In $110 / 222(50 \%)$ of all astrocytomas evaluated, a nuclear staining of annexin-1 was present, whereas only 6 out of 46 (13\%) oligodendrogliomas showed nuclear immunoreactivity (Table 2). Furthermore, the mean percentage of cells showing a nuclear staining was significantly higher in astrocytic neoplasms (mean: 12.1; 95 \% CI: 9.0-15.2) compared with their oligodendroglial counterparts (mean: 1.3 ; 95\% CI: 0-6.4, $P=0.002$ ). Controlling for each WHO grade, WHO grade III anaplastic astrocytoma $(35 / 52 ; 67 \%)$ and grade IV glioblastoma (41/67; 61\%) had a higher proportion of cells showing nuclear annexin-1 expression than grade I WHO pilocytic astrocytoma $(10 / 25 ; 40 \%)$ and grade II WHO diffuse astrocytomas (24/78; 31\%). Controlling for each WHO grade, there was no survival difference between nuclear annexin-1 expressing and negative tumors (Figure 5). WHO grade II oligodendrogliomas $(3 / 27 ; 11 \%)$ and grade III anaplastic oligodendrogliomas $(3 / 19 ; 16 \%)$ showed similar low levels of annexin-1 nuclear staining.

\section{Annexin-1 Expression Patterns in Various Neuroepithelial Tumors Differ between Tumor Entities}

We further examined annexin-1 expression in the less common neuroepithelial tumors. The 

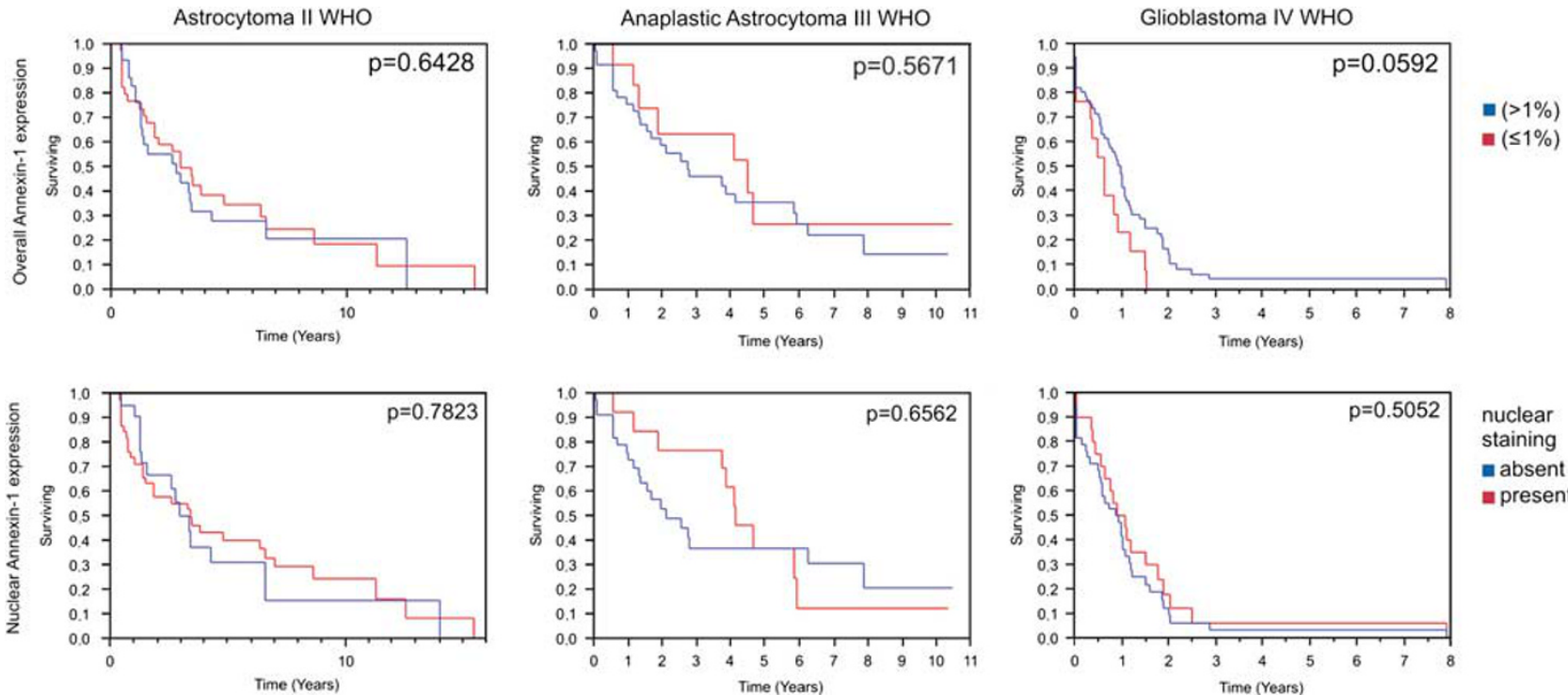

nuclear staining

absent a present

Figure 5 The overall survival for patients with astrocytomas of different grades of malignancy. The survival does not differ between extent of annexin-1-positive or annexin-1-negative $(\leq 1 \%)$ tumors (upper row) or nuclear annexin-1 expression (lower row).

epidemiologic data on these cases is also given in Table 1. In pilomyxoid astrocytomas (Figure 6a) the expression was observed in the cytoplasm of bipolar tumor cells, especially around tumor vessels sparing the surrounding mucinous matrix. Only few tumor cells also showed nuclear immunoreactivity. In pleomorphic xanthoastrocytomas (Figure 6b) there was a strong immunoreactivity for annexin-1 within the cytoplasm of tumor cells and in the eosinophilic granular bodies. A distinct nuclear staining was absent. Annexin-1-positive glial tumor cells with astrocytic morphology were observed in all gangliogliomas (Figure 6c), although the majority of glial tumor cells were immunonegative. Neuronal tumor cells and perivascular inflammatory infiltrates did not express annexin-1. The expression of annexin-1 in all central neurocytomas (including one extraventricular neurocytoma; Figure 6d) was restricted to endothelial cells, similar to the pattern observed in most oligodendroglial tumors. In contrast, choroid plexus papillomas (Figure 6e) showed strong membranous immunoreactivity, especially on the apical layer. In addition, the majority of cells showed a distinct nuclear staining. Immunoreactivity in subependymomas was heterogeneous but generally weaker than in classical ependymomas showing positive cells mainly in clusters close to microcysts (Figure 6f). In subependymal giant cell astrocytomas, there was a diffuse but a strong expression of annexin-1. A subset of tumor cells showed an additional membranous rim (Figure 6g). Myxopapillary ependymomas showed constant cytoplasmic immunoreactivity of the tumor cells (Figure 6h), although nuclear staining was observed in $<10 \%$ of the cells. In pilocytic astrocytomas, the annexin-1 expression was very heterogeneous but present in all tumor specimens observed. Especially, the long piloid cell processes, Rosenthal fibers and eosinophilic granular bodies showed strong immunoreactivity (Figure 6i). The oligodendroglial-like cells lacked annexin-1. Only occasionally annexin-1 was observed in the nuclei. In dysembryoplastic neuroepithelial tumors only a few of the cells within the glioneuronal element expressed cytoplasmic and nuclear immunoreactivity for annexin-1 whereas oligodendroglia-like clear cells and neuronal cells were negative (Figure 6j). This pattern is very similar to glial fibrillary acidic protein (GFAP) immunostaining. There was a striking divergent pattern of annexin-1 immunoreactivity in medulloblastomas from almost negative tumor specimens to a strong, nuclear and cytoplasmic immunoreactivity in $>90 \%$ of the tumor cells in one case. There was no specific pattern within the center of rosette formations, but in desmoplastic medulloblastomas, the reticulin-free areas of the so-called 'pale island' constantly lacked annexin-1 (Figure 6k). Cytoplasmic and nuclear annexin-1 expression was variable (scores 2 and 3, respectively) in both primitive neuroectodermal tumors of the central nervous system analyzed.

\section{Discussion}

The dysregulation in cancer is common among the annexin family, of which annexin-2 has been studied extensively. Beside non-small cell lung cancer and pancreatic tumors, an expression of annexin-2 has been shown in high-grade astrocytomas $^{22}$ and shows promigratory activity in glioma cells. ${ }^{23}$ As annexin-1 is a physiological substrate of EGFR, ${ }^{24}$ its role in gliomas is of great interest. Cell culture studies have shown that annexin-1 mediates 

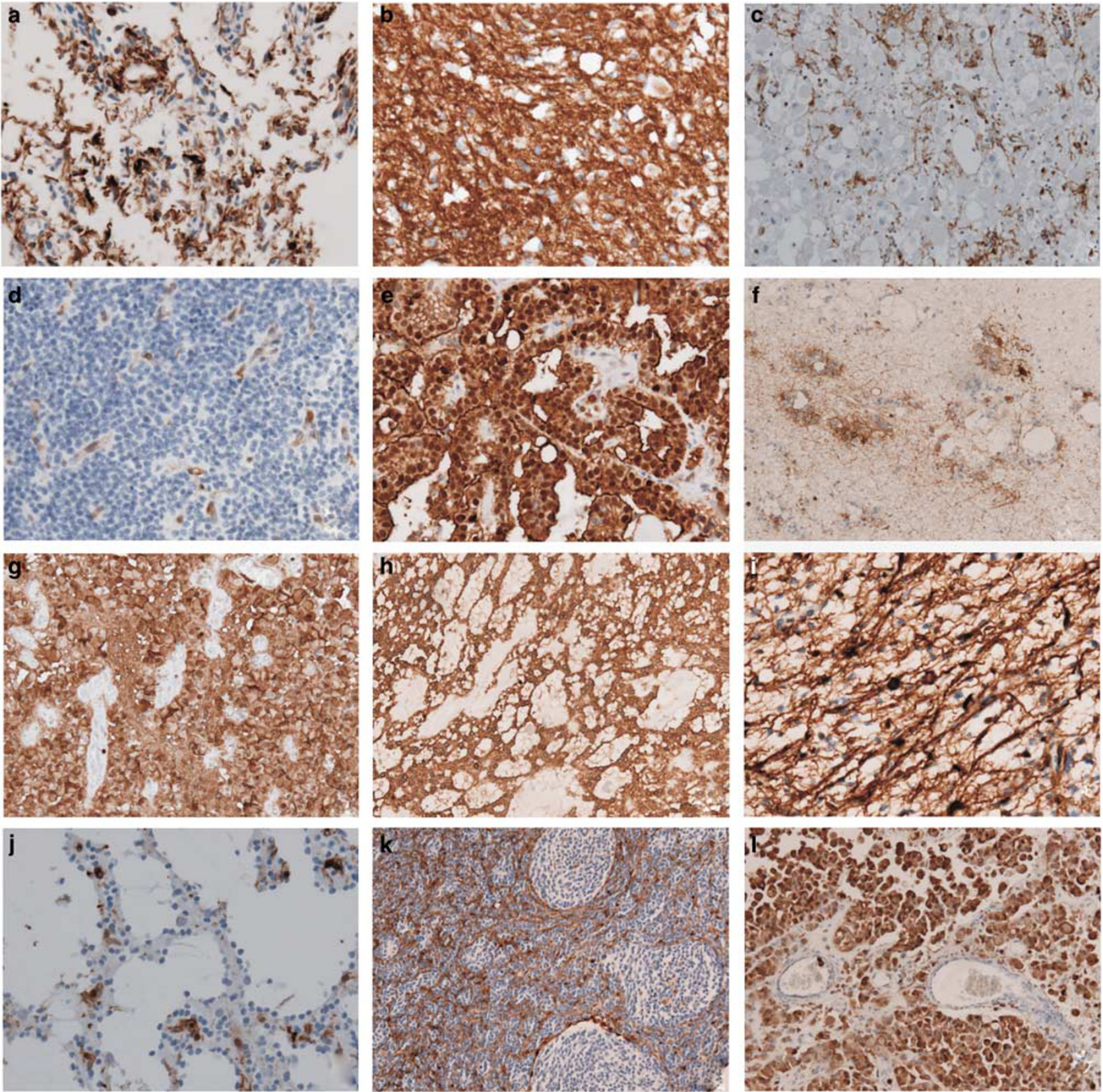

Figure 6 Annexin-1 expression in (a) pilomxyoid astrocytoma, (b) pleomorphic xanthoastrocytoma, (c) ganglioglioma, (d) central neurocytoma, (e) choroid plexus papilloma, (f) subependymoma, (g) subependymal giant cell astrocytoma, (h) myxopapillary ependymoma, (i) pilocytic astrocytoma, (j) dysembroplastic neuropithelial tumor, (k) desmoplastic medulloblastoma and (l) adenocarcinoma of the lung serving as additional positive control to Wilms' tumor.

the inhibitory effect on glucocorticoids on EGFstimulating cell growth through tyrosine phosphorylation. ${ }^{25}$ As an important mediator in glucocorticoid metabolism, the role of annexin-1 has been studied intensively in pituitary adenomas. ${ }^{5,26}$ A recent RNA microarray study has identified annexin- 1 as being one of the most overexpressed proteins in primary glioblastomas. ${ }^{27}$ The successful validation with western blot and RT-PCR data, allowed us to characterize annexin-1 protein expression in a large number of tumors using the tissue microrarray technique. In this study we are able to confirm that annexin-1 is abundantly upregulated in cytoplasm and nuclei in the tumor cells of human astrocytomas, whereas the expression of annexin-1 in normal white and grey matter is limited to some specialized structures, such as the ependymal lining, subependymal astrocytes, and a minority of choroid plexus epithelial cells. Not only did we note the annexin-1 expression in endothelial cells of cerebral vessels in normal brain, which is in accordance with a previous study, ${ }^{6,7}$ but we also observed that tumor vessels 
showed the same pattern. The functional role of annexin-1 in the vessels is not well understood, but a protective role is proposed. ${ }^{28}$ This consistent pattern serves as a good internal control in the otherwise immunonegative tumor specimens. In addition, we show that the annexin-1 expression levels significantly increase with WHO grade in diffuse astrocytomas. This suggests that the annexin-1 protein might be involved in malignant transformation of these tumors, although the full functional role therein remains unclear. In some neoplasms, annexin-1 is considered to act as a tumor suppressor protein. ${ }^{29}$ This is possibly due to regulation of the ERK/MAPK, as annexin-1 inhibits cell proliferation by ERKmediated disruption of the actin cytoskeleton and by ablation of cyclin D1 protein expression. ${ }^{9}$ Taken together, the expression of annexin-1 in cancers is highly tissue specific. Although it is downregulated in thyroid $^{30}$ and prostrate ${ }^{31}$ cancer, upregulated annexin-1 levels are observed in hairy cell leukemia, esophageal and pancreatic adenocarcinoma (reviewed by Mussunoor and Murray ${ }^{32}$ ). Thus, annexin-1 expression needs to be determined for each tumor type individually. This might explain why annexin-1 is also strongly upregulated in several specialized low-grade neoplasms. This is most notable in pilocytic astrocytomas, as these tumors arise on a genetic background that is different from diffuse astrocytomas. ${ }^{16}$

We have shown that annexin-1 expression levels are higher in primary glioblastomas than in secondary glioblastomas, which result from malignant astrocytoma progression. Similarly, the annexin-1 substrate EGFR, ${ }^{14,15}$ which transduces proliferative activity, is overexpressed in most primary glioblastomas. ${ }^{33}$ In contrast, EGFR amplification is rarely observed in secondary glioblastomas. ${ }^{17,18}$ The similar upregulation suggests that the interaction between annexin-1 and EGFR might also exist in vivo, which has been shown in vitro. ${ }^{14,15}$ Further studies are needed to clarify this issue. In contrast to primary glioblastomas, ${ }^{34}$ EGFR is absent or very low in pilocytic astrocytomas. ${ }^{35}$ Thus, in pilocytic astrocytomas, the increased expression levels of annexin-1 do not parallel with higher EGFR levels, indicating an additional function of annexin-1 besides serving as a substrate for EGFR.

The number of tumor cells showing nuclear annexin-1 expression increased with the grade of malignancy in astrocytomas. This is in line with previously reported nuclear expression levels of FABP7, which is downstream of the Ras-independent EGFR signaling pathway, in pilocytic astrocytomas and glioblastomas. ${ }^{36}$ However, we were not able to observe any association with nuclear annexin-1 and survival.

Furthermore, we show that ependymomas, as well as a few other neuroepithelial tumor entities, also express annexin-1 at high levels, whereas annexin-1 is significantly reduced in oligodendrogliomas. In this context, it is interesting that ependymomas show a frequent gain of function on the chromosomal arm 9q, where the annexin-1 gene is located, ${ }^{37}$ whereas chromosome 9 losses have been reported in some oligodendroglial tumors. ${ }^{38}$ Nuclear staining of annexin-1 in oligodendroglial tumor cells did not exceed $7 \%$ of all tumor cells. In contrast, one-third $(33 \%)$ of all glioblastomas had a nuclear staining in $>7 \%$ of all the tumor cells. We have found this different nuclear staining pattern to be helpful in distinguishing astrocytomas and oligodendrogliomas, especially as the majority of oligodendroglial tumor cells are also devoid of cytoplasmic annexin-1 (Figure 4b). Similar expression patterns have been reported for Nogo A, ${ }^{39}$ YKL- $40^{40}$ and Wilms' tumor protein WT1. ${ }^{19}$ In doubtful cases, or mixed glial neoplasms, a panel of the above-mentioned markers might be helpful in determining the tumor origin or the amount of different components.

As cell culture experiments have shown that glucocorticoids are capable of mitigating tumor growth, determination of annexin-1 expression levels in gliomas using methods of immunohistochemisty might become useful. Further studies in correlating annexin-1 expression and steroid response of tumors beforehand are needed for verifying the cell culture experiments in vivo.

In conclusion, the upregulation of annexin-1 (and also of annexin-2, which has been reported previously) suggest that they may have a role in the development and/or progression of astrocytomas.

\section{Acknowledgement}

We thank Thai Dung Nguyen for help with additional immunostainings and Melanie L. Hart for her proofreading this paper.

\section{Disclosure/conflict of interest}

The authors declare no conflict of interest.

\section{References}

1 Lim LH, Pervaiz S. Annexin 1: the new face of an old molecule. FASEB J 2007;21:968-975.

2 Smith PD, Moss SE. Structural evolution of the annexin supergene family. Trends Genet 1994;10: 241-246.

3 Christmas P, Callaway J, Fallon J, et al. Selective secretion of annexin 1, a protein without a signal sequence, by the human prostate gland. J Biol Chem 1991;266:2499-2507.

4 Solito E, McArthur S, Christian H, et al. Annexin A1 in the brain-undiscovered roles? Trends Pharmacol Sci 2008;29:135-142.

5 Johnson MD, Gray ME, Pepinsky RB, et al. Lipocortin-1 immunoreactivity in the human pituitary gland. J Histochem Cytochem 1990;38:1841-1845.

6 Eberhard DA, Brown MD, VandenBerg SR. Alterations of annexin expression in pathological neuronal and 
glial reactions. Immunohistochemical localization of annexins II I (p36 and p11 subunits), IV, and VI in the human hippocampus. Am J Pathol 1994;145:640-649.

7 Dreier R, Schmid KW, Gerke V, et al. Differential expression of annexins I, II and IV in human tissues: an immunohistochemical study. Histochem Cell Biol 1998;110:137-148.

8 Probst-Cousin S, Kowolik D, Kuchelmeister K, et al. Expression of annexin-1 in multiple sclerosis plaques. Neuropathol Appl Neurobiol 2002;28:292-300.

9 Alldridge LC, Bryant CE. Annexin 1 regulates cell proliferation by disruption of cell morphology and inhibition of cyclin D1 expression through sustained activation of the ERK1/2 MAPK signal. Exp Cell Res 2003;290:93-107.

10 D'Acquisto F, Merghani A, Lecona E, et al. Annexin-1 modulates T-cell activation and differentiation. Blood 2007;109:1095-1102.

11 Solito E, de Coupade C, Canaider S, et al. Transfection of annexin 1 in monocytic cells produces a high degree of spontaneous and stimulated apoptosis associated with caspase-3 activation. Br J Pharmacol 2001;133:217-228.

12 Alldridge LC, Harris HJ, Plevin R, et al. The annexin protein lipocortin 1 regulates the MAPK/ERK pathway. J Biol Chem 1999;274:37620-37628.

13 De BK, Misono KS, Lukas TJ, et al. A calciumdependent 35-kilodalton substrate for epidermal growth factor receptor/kinase isolated from normal tissue. J Biol Chem 1986;261:13784-13792.

14 White IJ, Bailey LM, Aghakhani MR, et al. EGF stimulates annexin 1-dependent inward vesiculation in a multivesicular endosome subpopulation. EMBO J 2006;25:1-12.

15 Gerke V, Moss SE. Annexins: from structure to function. Physiol Rev 2002;82:331-371.

16 Louis DN, Ohgaki H, Wiestler OD, et al. (eds). Pathology \& Genetics: WHO Classification of Tumours of the Central Nervous System. International Agency for Research on Cancer Press: Lyon, 2007.

17 Ohgaki H, Kleihues P. Genetic pathways to primary and secondary glioblastoma. Am J Pathol 2007;170:1445-1453.

18 Watanabe K, Tachibana O, Sata K, et al. Overexpression of the EGF receptor and p53 mutations are mutually exclusive in the evolution of primary and secondary glioblastomas. Brain Pathol 1996;6:217-223.

19 Schittenhelm J, Beschorner R, Simon P, et al. Diagnostic value of WT1 in neuroepithelial tumours. Neuropathol Appl Neurobiol 2009;35:69-81.

20 Landis JR, Koch GG. The measurement of observer agreement for categorical data. Biometrics 1977;33: 159-174.

21 Mittelbronn M, Capper D, Bunz B, et al. De novo erythropoietin receptor (EPO-R) expression in human neoplastic glial cells decreases with grade of malignancy but is favourably associated with patient survival. Neuropathol Appl Neurobiol 2007;33:299-307.

22 Roseman BJ, Bollen A, Hsu J, et al. Annexin II marks astrocytic brain tumors of high histologic grade. Oncol Res 1994;6:561-567.

23 Tatenhorst L, Rescher U, Gerke V, et al. Knockdown of annexin 2 decreases migration of human glioma cells in vitro. Neuropathol Appl Neurobiol 2006;32:271-277.

24 Pepinsky RB. Phosphorylation of lipocortin-1 by the epidermal growth factor receptor. Methods Enzymol 1991;198:260-272.
25 Croxtall JD, Gilroy DW, Solito E, et al. Attenuation of glucocorticoid functions in an Anx-A1-/- cell line. Biochem J 2003;371:927-935.

26 Mulla A, Christian HC, Solito E, et al. Expression, subcellular localization and phosphorylation status of annexins 1 and 5 in human pituitary adenomas and a growth hormone-secreting carcinoma. Clin Endocrinol 2004;60:107-119.

27 Ruano Y, Mollejo M, Camacho FI, et al. Identification of survival-related genes of the phosphatidylinositol 3'-kinase signaling pathway in glioblastoma multiforme. Cancer 2008;112:1575-1584.

28 Herbert SP, Odell AF, Ponnambalam S, et al. The confluence-dependent interaction of cytosolic phospholipase A2-alpha with annexin A1 regulates endothelial cell prostaglandin E2 generation. J Biol Chem 2007;282:34468-34478.

29 Shen D, Nooraie F, Elshimali Y, et al. Decreased expression of annexin A1 is correlated with breast cancer development and progression as determined by a tissue microarray analysis. Human Pathol 2006;37: 1583-1591.

30 Petrella A, Festa M, Ercolino SF, et al. Annexin-1 downregulation in thyroid cancer correlates to the degree of tumor differentiation. Cancer Biol Ther 2006;5:643-647.

31 Paweletz CP, Ornstein DK, Roth MJ, et al. Loss of annexin 1 correlates with early onset of tumorigenesis in esophageal and prostate carcinoma. Cancer Res 2000;60:6293-6297.

32 Mussunoor S, Murray GI. The role of annexins in tumour development and progression. J Pathol 2008;216:131-140.

33 Ohgaki H, Dessen P, Jourde B, et al. Genetic pathways to glioblastoma: a population-based study. Cancer Res 2004;64:6892-6899.

34 Muracciole X, Romain S, Dufour H, et al. PAI-1 and EGFR expression in adult glioma tumors: toward a molecular prognostic classification. Int J Radiat Oncol Biol Phys 2002;52:592-598.

35 Huang H, Hara A, Homma T, et al. Altered expression of immune defense genes in pilocytic astrocytomas. J Neuropathol Exp Neurol 2005;64:891-901.

36 Liang Y, Bollen AW, Aldape KD, et al. Nuclear FABP7 immunoreactivity is preferentially expressed in infiltrative glioma and is associated with poor prognosis in EGFR-overexpressing glioblastoma. BMC Cancer 2006;6:97.

37 Zheng PP, Pang JC, Hui AB, et al. Comparative genomic hybridization detects losses of chromosomes 22 and 16 as the most common recurrent genetic alterations in primary ependymomas. Cancer Genet Cytogenet 2000;122:18-25.

38 Kitange G, Misra A, Law M, et al. Chromosomal imbalances detected by array comparative genomic hybridization in human oligodendrogliomas and mixed oligoastrocytomas. Genes Chromosomes Cancer 2005;42:68-77.

39 Kuhlmann T, Gutenberg A, Schulten HJ, et al. Nogo-a expression in glial CNS tumors: a tool to differentiate between oligodendrogliomas and other gliomas? Am J Surg Pathol 2008;32:1444-1453.

40 Rousseau A, Nutt CL, Betensky RA, et al. Expression of oligodendroglial and astrocytic lineage markers in diffuse gliomas: use of YKL-40, ApoE, ASCL1, and NKX2-2. J Neuropathol Exp Neurol 2006;65:1149-1156. 